\title{
Reference-based annotation of single cell transcriptomes identifies a profibrotic macrophage niche after tissue injury
}

Dvir Aran ${ }^{1 \S}$, Agnieszka P. Looney ${ }^{2 \S}$, Leqian Liu ${ }^{3 \S}$, Valerie Fong ${ }^{2}$, Austin Hsu ${ }^{4}$, Paul J. Wolters $^{2}$, Adam Abate ${ }^{3}$, Atul J. Butte ${ }^{1}$, Mallar Bhattacharya ${ }^{2 *}$

1 Institute for Computational Health Sciences, University of California, San Francisco, California, 94158, USA.

${ }^{2}$ Division of Pulmonary, Critical Care, Allergy, and Sleep, Department of Medicine, University of California, San Francisco, USA.

3 Department of Bioengineering and Therapeutic Sciences, University of California, San Francisco, USA.

${ }^{4}$ Gladstone Institute of Cardiovascular Disease, San Francisco, CA 94158, USA.

* Correspondence: mallar.bhattacharya@ucsf.edu

$\S$ These authors contributed equally. 


\begin{abstract}
Myeloid cells localize to peripheral tissues in a wide range of pathologic contexts. However, appreciation of distinct myeloid subtypes has been limited by the signal averaging inherent to bulk sequencing approaches. Here we applied single-cell RNA sequencing (scRNA-seq) to map cellular heterogeneity in lung fibrosis induced by bleomycin injury in mice. We first developed a computational framework that enables unbiased, granular cell-type annotation of scRNA-seq. This approach identified a macrophage subpopulation that was specific to injured lung and notable for high expression of Cx3cr1+ and MHCII genes. We found that these macrophages, which bear a gene expression profile consistent with monocytic origin, progressively acquire alveolar macrophage identity and localize to sites of fibroblast accumulation. Probing their functional role, in vitro studies showed a trophic effect of these cells on fibroblast activation, and ablation of $C x 3 \mathrm{cr} 1$-expressing cells suppressed fibrosis in vivo. We also found by gene set analysis and immunofluorescence that markers of these macrophages were upregulated in samples from patients with lung fibrosis compared with healthy controls. Taken together, our results uncover a specific pathologic subgroup of macrophages with markers that could enable their therapeutic targeting for fibrosis.
\end{abstract}

\title{
Main
}

Fibrosing diseases are a major cause of mortality and, whether toxin-related, infectious, autoimmune, or idiopathic, can affect any organ ${ }^{1}$. Lung fibrosis is a particularly vexing clinical problem because of the lack of effective therapies and a poor understanding of its etiology. The most prevalent form, idiopathic pulmonary fibrosis, has a median survival of only 3 years, and approved treatments are limited ${ }^{2}$. With respect to pathogenesis, much attention has focused on fibroblast activation, given the central role of fibroblasts in the deposition of matrix proteins such as collagen ${ }^{3-6}$. However, the causes of fibroblast activation and proliferation are not fully understood.

The healthy adult lung contains both a self-renewing population of embryonically-derived macrophages known as alveolar macrophages and a separate population of interstitial macrophages present near the larger airways and in the lung interstitium ${ }^{7,8}$. In lung fibrosis models, macrophages expand in number and have been found to be profibrotic overall based on studies of broad-based ablation, such as with clodronate and pan-macrophage or developmental knockouts ${ }^{9,10}$. We used scRNA-seq to capture macrophage heterogeneity in the fibrotic phase of bleomycin injury and thus to enhance detection of functional subsets in fibrogenesis.

To facilitate analysis of mixed populations present in cell suspensions derived from whole lung, we developed SingleR (Single Cell Recognition), a reference-based computational tool that enables unbiased annotation of scRNA-seq. Whereas the prevalent approach in scRNA-seq has been to classify clusters of cells using marker genes, manual annotation suffers from subjectivity and low resolution. Furthermore, traditional markers have often been developed for flow cytometry based on their robust protein expression; however, the expression of these markers at the mRNA level may not support their use for transcriptomic approaches, particularly given the low detection and frequent dropout with scRNA-seq. SingleR overcomes these limitations by assigning cell-type identity to single cells by unbiased 
bioRxiv preprint doi: https://doi org/10.1101/284604; this version posted March 22, 2018. The copyright holder for this preprint (which was not certified by peer review) is the author/funder, who has granted bioRxiv a license to display the preprint in perpetuity. It is made available under aCC-BY-NC-ND 4.0 International license.

comparison to reference datasets of pure cell types sequenced by microarray or RNA-seq. The SingleR pipeline (Figure 1a and Supplementary Information) first correlates the single cell transcriptome against selected reference datasets. SingleR then iteratively fine-tunes the correlations by reanalyzing the top correlated cell types with a reduced set of genes that differentiate between them, allowing granular annotation of cell type.

Figure 1 Annotation of scRNA-seq by reference datasets reveals disease-associated macrophages in lung fibrosis. a Schematic of SingleR, a protocol for cell-type annotation by reference to transcriptomes of pure cell types (details in Supplementary Information). $\mathbf{b}$, SingleR applied to a published scRNA-seq dataset of fibroblast and bone marrow-derived dendritic cells (BMDC) ${ }^{11}$ Top left: Original identities indicated on t-SNE plot. Top right: Heatmap of SingleR scores for top correlated cell types. Bottom left: SingleR annotation of cell identity indicated on t-SNE plot. Bottom right: Expression in single cells of genes derived by differential expression analysis of published microarray data from bone marrow-derived, GM-CSF-cultured macrophages (GM-Macs) and DCs (GM-DCs) $)^{15}$ confirms SingleR annotations. c-e, t-SNE plots of single-cell suspensions from mouse whole lung sequenced by Dropseq and color-coded for experimental condition (c), annotated by SingleR with the ImmGen reference database (d), and annotated by SingleR with lung-specific myeloid datasets ${ }^{24,25}$ (e). Data shown pool replicates $(n=3$ mice for bleomycin, $n=5$ mice for control).
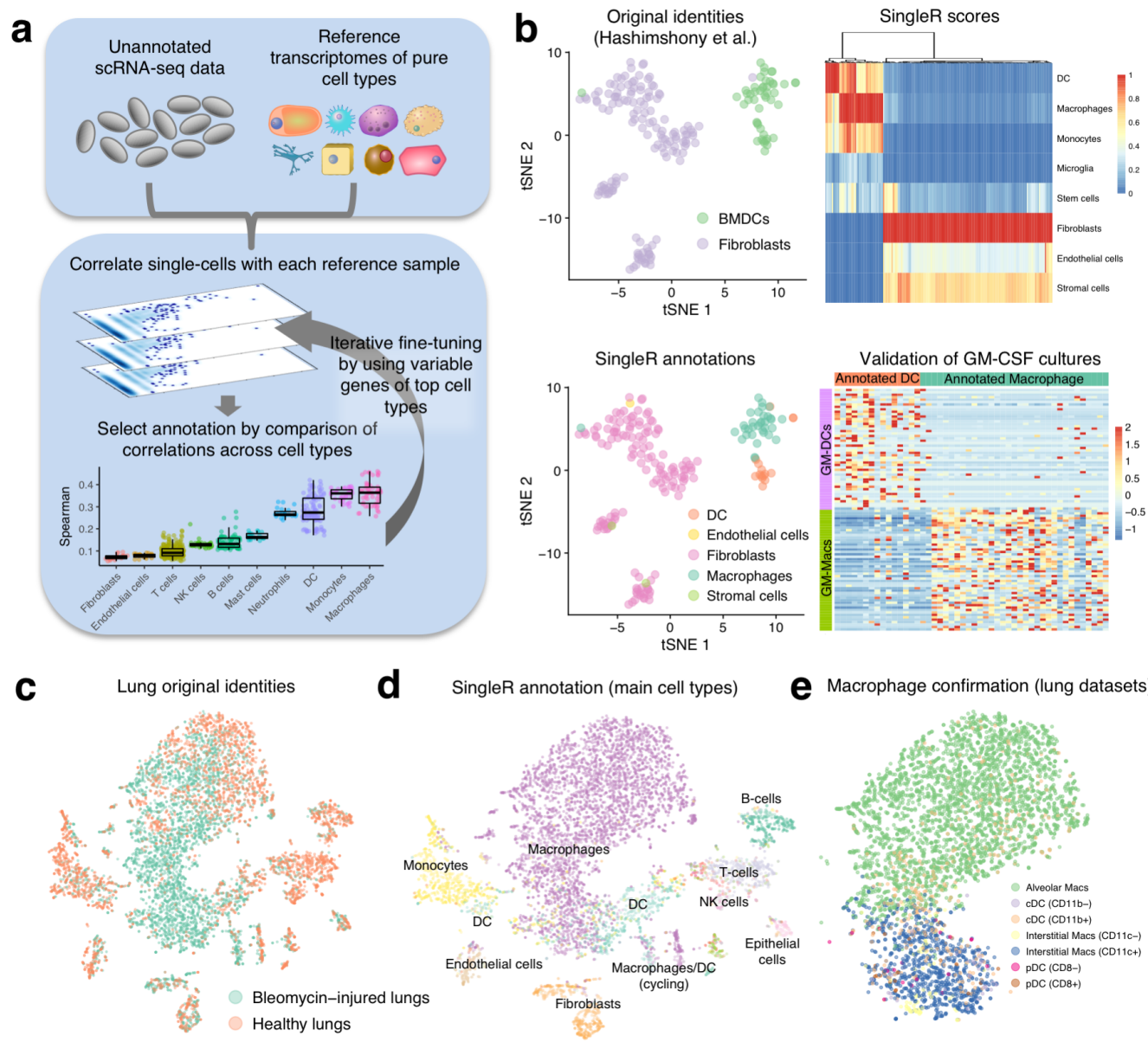

SingleR annotation (main cell types)

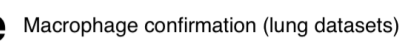

We first validated SingleR with several publicly available scRNA-seq datasets. For example, we studied scRNA-seq datasets of mouse bone marrow-derived dendritic cells (BMDC) and mouse fibroblasts published by Hashimshony et al. ${ }^{11}$ (Figure 1b). T-distributed stochastic neighbor embedding (tSNE) analysis of gene expression revealed separate clusters of fibroblasts and 
BMDCs conforming to their specified identities. We then applied SingleR using the ImmGen database of 830 microarray samples encompassing a broad range of pure mouse cell types ${ }^{12}$ as the reference library for unbiased annotation. Superimposing these annotations on the tSNE plot confirmed fibroblast identity but revealed, surprisingly, that 33 of the 48 BMDCs were macrophages. Of note, cells in the study were cultured with GM-CSF and sorted on $\mathrm{CD} 11 \mathrm{c}$ for isolation; however, a recent study has shown that this procedure, which had been widely used in studies of DCs ${ }^{13,14}$, is not exclusive for DCs but also captures macrophages ${ }^{15,16}$. Comparative analysis using microarray data from this study confirmed segregation according to macrophage or DC lineage, in accordance with SingleR. These results show that SingleR enhances cell type annotation of scRNA-seq to a higher and more accurate resolution. Similar analyses for other datasets with comparisons of SingleR against alternative annotation methods ${ }^{17,18}$ as well as its application to $>50$ publicly available human and mouse scRNA-seq datasets are presented in Supplementary Information and in a dedicated web app http://comphealth.ucsf.edu/SingleR/.

We next applied SingleR to enhance analysis of transcriptomic change in lung fibrosis across multiple cell types by Drop-seq analysis of collagenase-digested whole mouse lungs, either at baseline or two weeks after bleomycin injury ${ }^{19}$. Altogether we sequenced 42,000 single-cells across five experiments, with 8,366 containing at least 500 non-zero genes, and used the Seurat package $^{20}$ to perform tSNE for visualization of cellular clusters (Figure 1c, Supplementary Table 1, and Supplementary Figure 1). Using SingleR with the ImmGen database, we mapped the annotations to the tSNE plot (Figure 1d and Supplementary Figure 2). This analysis revealed a disproportionately large number of cells from bleomycin-injured lungs in a large central cluster (Figure 1c) that was annotated as macrophages (Figure 1d). Since macrophages and dendritic cells are known to have similar transcriptomic profiles under certain circumstances $^{21-23}$, we repeated SingleR analysis using published RNA-seq datasets of mouse lung macrophages and dendritic cells ${ }^{24,25}$ for greater lung specificity. This analysis confirmed a predominance of lung macrophages as opposed to dendritic cells (Figure 1e and Supplementary Figure 3).

Two major annotations were resolved by this analysis: alveolar and interstitial macrophages. These annotations were strongest at the poles of our tSNE cluster, suggesting the possibility that there might be a continuum of gene expression between these macrophage cell types. Therefore, we quantified each cell by similarity analysis to the RNA-seq datasets of alveolar and interstitial macrophages ${ }^{25}$. This analysis revealed cells with intermediate gene expression between the two cell types (Figure 2a and Supplementary Figure 4). To better understand this intermediate cluster, we next used SingleR for unsupervised hierarchical subclustering of macrophages based on differential annotation using the full range of cell types within the ImmGen database and, consistent with our gradient analysis, identified three distinct groups: alveolar macrophages (C1), interstitial macrophages (C3), and an intermediate cluster of cells that were mapped by SingleR to both alveolar and non-alveolar macrophage reference datasets (C2; Figure 2b and Supplementary Figure 5). 
bioRxiv preprint doi: https://doi.org/10.1101/284604; this version posted March 22, 2018. The copyright holder for this preprint (which was not certified by peer review) is the author/funder, who has granted bioRxiv a license to display the preprint in perpetuity. It is made available under aCC-BY-NC-ND 4.0 International license.

\begin{abstract}
Figure 2 Macrophages expressing $\mathrm{C} \times 3 \mathrm{cr} 1$ and $\mathrm{MHCll}$ localize to sites of fibroblast accumulation after lung injury. a, Quantification of similarity in gene expression of individual cells to bulk RNA-seq profiles of alveolar macrophages (AM) and $\mathrm{CD} 11 \mathrm{c}+$ interstitial macrophages $(\mathrm{IM})^{25}$. b. Subclustering of macrophages based on SingleR annotation by reference to the ImmGen database. c, Heatmap of genes differentially expressed between $\mathrm{C} 1$ and $\mathrm{C} 3$ with examples superimposed on the tSNE plot. d, Percentage of cells in each cluster that express genes in c (differentially expressed between C1 and C3). $45 \%$ of C2 cells express at least a third of $\mathrm{C} 1$ genes and at least a third of $\mathrm{C} 3$ genes. e, Heatmap of the most differentially expressed genes from bulk RNAseq of alveolar macrophages (SiglecF+CD11C+) sorted on MHCII-low at baseline and both MHC-low and MHCIl-high at two time points after bleomycin ( $n=2$ mice for baseline, $n=3$ mice at 2 weeks, $n=3$ mice at 4 weeks). $\mathbf{f - g}$, Lineage tracing of lung cells in Cx3cr1-CreERT2 / Rosa26 $6_{\text {loxp }}$ STOP $_{\text {loxp }}$-TdTomato mice with tamoxifen administration before and after injury followed by flow cytometry, with contour plots and box plots depicting values for TdTomato+ cells ( $f, n=3$ mice for each group, mean is marked), and immunofluorescence ( $\mathbf{g}$, representative image is shown, $n=3$ mice).
\end{abstract}

a

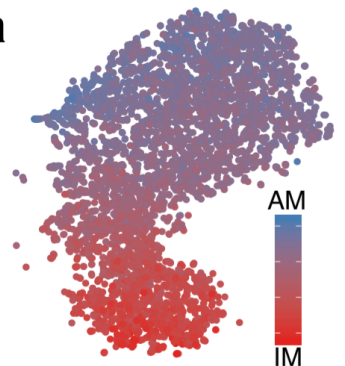

b

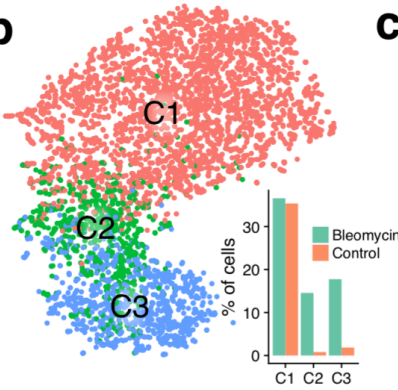

d

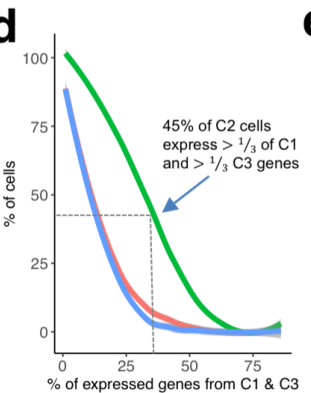

e

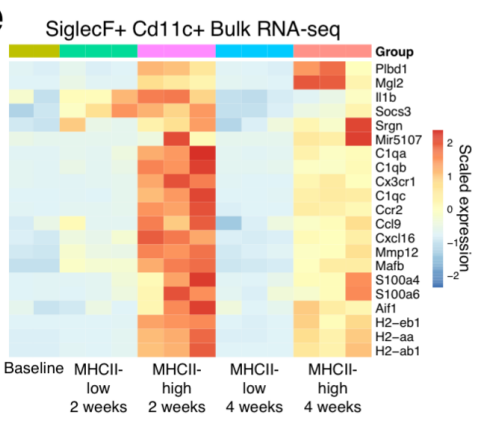

$\mathbf{f}$

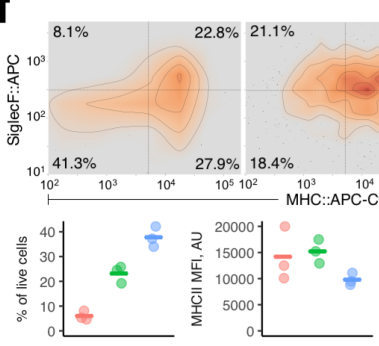

C
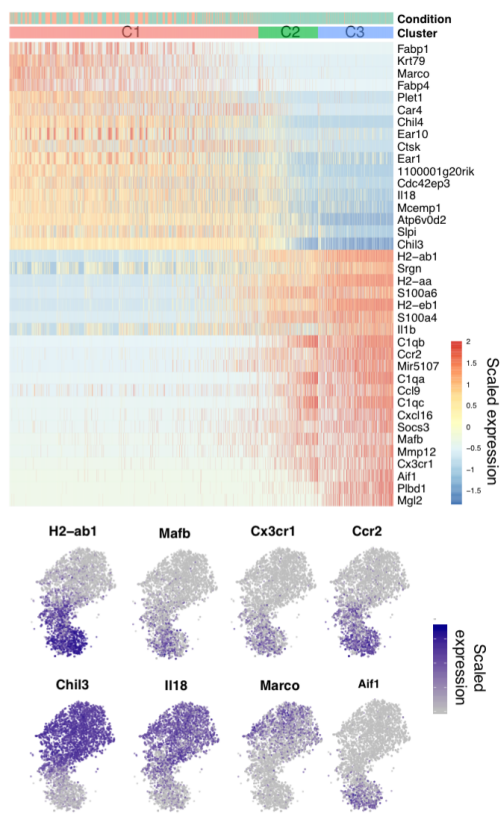
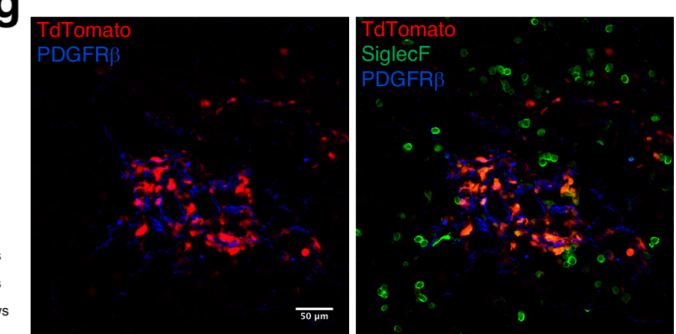

Notably, both the intermediate subcluster C2 and C3 were highly enriched for cells from bleomycin-induced fibrosis. Differential gene expression between $\mathrm{C} 1$ and $\mathrm{C} 3$ and yielded 38 genes with $\log _{2}$ fold change $>1.5$ (Figure 2c and Supplementary Table 2). Remarkably, a high percentage of cells from $\mathrm{C} 2$ had high expression of genes from both $\mathrm{C} 1$ and $\mathrm{C} 3$ (Figure 2d), suggesting that $\mathrm{C} 2$ represents a transitional state unique to the disease model and intermediate between $\mathrm{C} 1$ and C3. Moreover, Cx3cr1, Ccr2, Mafb, and MHCII genes were common to $\mathrm{C} 2$ and $\mathrm{C} 3$ and are consistent with macrophages of monocytic origin, as previously reported in bleomycin-induced lung fibrosis ${ }^{10,26}$. To confirm these findings for $\mathrm{C} 2$, we sorted alveolar macrophages (SiglecF $+\mathrm{CD} 11 \mathrm{c}+$ ) after injury by MHCII-low and MHC-high expression to distinguish the $\mathrm{C} 1$ and $\mathrm{C} 2$ subclusters, respectively. MHCII-high 
cells compared with MHCII-low shared almost all the genes identified by scRNA-seq (Figure 2e and Supplementary Figure 6a).

We next undertook studies to probe the functional niche of these macrophages in vivo. Since Cx3cr1 was expressed in both subclusters $\mathrm{C} 2$ and $\mathrm{C} 3$, we took advantage of the tamoxifeninducible allele Cx3cr1-CreERT2 ${ }^{27}$ crossed with Rosa26-loxp-STOP-loxp-TdTomato for lineage tracing. Tamoxifen induction of healthy mice showed that a minority of lung cells, likely airway and interstitial macrophages, express $C x 3 c r 1$ at baseline (Figure $2 \mathrm{f}$ and Supplementary Figure 6b). After injury, we observed an increase in Cx3cr1-lineage cells (CLCs). Furthermore, these cells progressively decreased expression of MHCII and increased expression of SiglecF, consistent with a transition of CLCs toward alveolar macrophage identity. Also consistent with a transition is the fact that, by bulk RNA-seq of alveolar macrophages (Figure 2e), the expression of $\mathrm{C} 2$ and $\mathrm{C} 3$ genes that were elevated at 2 weeks decreased toward baseline levels at 4 weeks after injury.

Surprisingly, we noted by confocal microscopy that CLCs were in direct contact with fibroblasts clustering at regions of injury and marked by expression of Pdgfrß (Figure 2g). Consistent with our scRNA-seq analysis, these CLCs also expressed the alveolar macrophage marker SiglecF, whereas non-lineage traced SiglecF+ macrophages (corresponding to the C1 subcluster) were absent from the fibrotic niche. Noting this localization of CLCs to sites of fibroblast accumulation, where scar forms in the model, we wondered whether these macrophages could provide trophic support for fibroblasts. This hypothesis is supported by recent in vitro evidence of the dependence of fibroblasts on macrophage-derived platelet derived growth factor (PDGF) ${ }^{28}$. Importantly, Pdgfa was upregulated in our bulk RNAseq analysis of activated (MHCII-high) alveolar macrophages after injury (Figure 3a). To test whether CLCs support fibroblast migration or proliferation via Pdgfa, we collected conditioned medium from sorted alveolar macrophages and tested its effect on cultured fibroblasts in an in vitro gap closure assay. Conditioned media from MHCII-high alveolar macrophages sorted from injured lung significantly enhanced gap closure by $3 \mathrm{~T} 3$ fibroblasts compared with MHCII-low conditioned media, an effect that was inhibited by antibody blockade of PDGF-AA (Figure 3b). To test the function of CLCs in vivo, we employed Diphtheria Toxin A-mediated ablation of cells expressing Cx3cr1 during the fibrotic phase of bleomycin lung injury (days 8 through 21). Remarkably, CLC ablation decreased bleomycininduced lung fibrosis by hydroxyproline assay (Figure 3c and Supplementary Figure 7). These results support a profibrotic effect of CLCs after injury and suggest that CLCs induce fibrosis via a trophic effect on fibroblasts.

Finally, we tested the relevance of CLCs to human disease. Immunofluorescence of samples from patients with idiopathic lung fibrosis as opposed to healthy controls was notable for expression of MAFB, a marker unique to CLCs (Figure 2c), in CD68+ macrophages (Figure $3 \mathrm{~d})$. Furthermore, gene set analysis using human orthologues of murine genes identified in our study confirmed decreased alveolar macrophage gene expression and increased CX3CR1 and MHC II genes in a published microarray dataset from patients with idiopathic pulmonary fibrosis compared to healthy controls (Figure $3 \mathrm{e})^{29}$. 
bioRxiv preprint doi: https://doi org/10.1101/284604; this version posted March 22, 2018. The copyright holder for this preprint (which was not certified by peer review) is the author/funder, who has granted bioRxiv a license to display the preprint in perpetuity. It is made available under aCC-BY-NC-ND 4.0 International license.

\begin{abstract}
Figure 3 C $\times 3 c r 1$-derived macrophages are necessary for lung fibrosis and are upregulated in human disease. a, Pdgfa expression by bulk RNAseq of SiglecF+CD11c+ lung macrophages sorted after bleomycin injury. Mean is marked. Wald test pvalue is presented. $\mathbf{b}, 3 \mathrm{~T} 3$ mouse fibroblast migration assay in presence of conditioned media (CM) from lung macrophages sorted by high and low MHCII expression 2 weeks after lung injury, with and without PDGF-AA blocking antibody ( $\mathrm{Ab} ; \mathrm{n}=6$ mice). Each data point corresponds to a separate assay. c, Lung collagen content measured by hydroxyproline assay 21 days after injury with and without macrophage ablation during the fibrotic period (day 8 onward) in Cx3cr1-CreERT2 $\times$ Rosa26 $6_{\text {loxp }}$ STOP Ioxp $_{\text {oxptheria Toxin }}$ A mice ( $n=7$ mice in each group). $d$, Representative immunofluorescence images of lung sections taken from human fibrotic and control lung with quantitation ( 10 fields of view per sample, $n=3$ fibrotic and 3 normal lung samples). $\mathrm{SH}=$ second harmonic imaging of collagen. e, Expression of human orthologues of the ImmGen alveolar (AM) signature, MHCII genes, and CX3CR1 in bulk RNAseq samples from patient lung biopsy specimens ${ }^{29}$. Mean is shown in all boxplots, and Wilcoxon text p-values are presented. ${ }^{* *} p<0.01 .{ }^{* * *} p<0.001$.
\end{abstract}

a
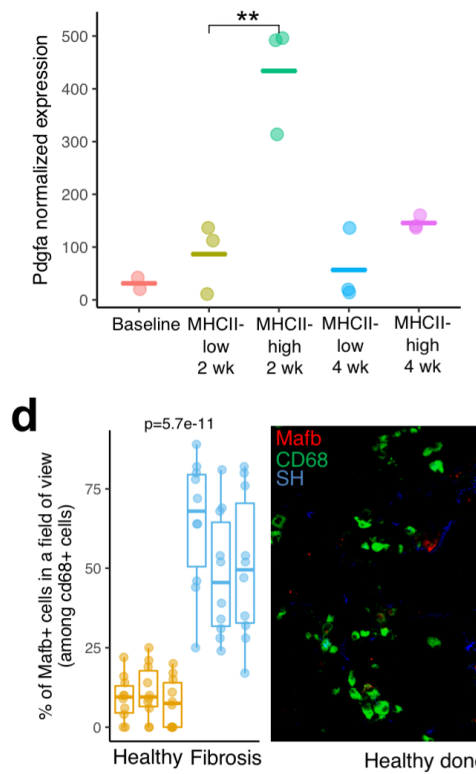

b

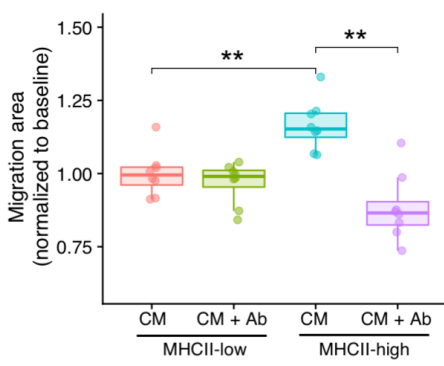

C

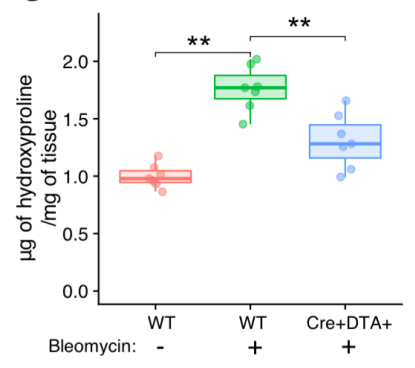

e

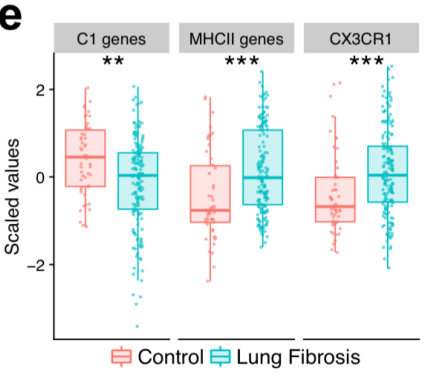

In summary, this study reveals a profibrotic macrophage subpopulation that localizes to sites of fibrotic scar with activating effects on the lung mesenchyme. Furthermore, the identification of specific markers for this group, including CX3CR1, could be used for therapeutic targeting in fibrosis. We also demonstrate the value of using reference-based annotation of single cell datasets in order to map the heterogeneity of canonical cell types such as macrophages, enhancing differentiation of transcriptomically unique subclusters within the data. Coupled with lineage tracing and functional studies, this approach should serve as a broadly applicable platform for study of specific cellular compartments in health and disease.

\title{
Methods
}

\section{Mice}

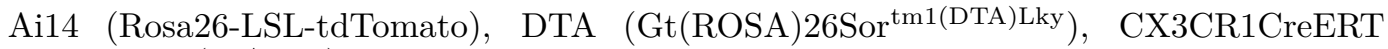

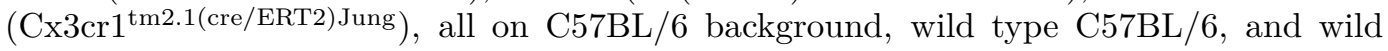
type 129S1 mice were obtained from the Jackson Laboratory. Strain-appropriate controls 
were used in all experiments, and genotyping of the mice was performed by PCR. CX3CR1-CreERT2 mice were administered $2 \mathrm{mg}$ tamoxifen (Sigma) via IP injection every other day for Cre induction. Mice were maintained in specific-pathogen-free conditions in the Animal Barrier Facility of the University of California, San Francisco. All animal experiments were approved by the Institutional Animal Care and Use Committee of the University of California, San Francisco.

\section{Microfabrication of Devices}

The PDMS Drop-seq droplet microfluidic device is fabricated with standard soft lithography techniques. Photoresist masters are created by spinning a layer of photoresist SU-8 (Microchem) onto a 3 inch silicon wafer (University Wafer), then baking at $95^{\circ} \mathrm{C}$ for 20 minutes. Then, the photoresist is subjected to 3 minutes ultraviolet exposure over Drop-seq ${ }^{19}$ photolithography masks (CAD/Art Services) printed at 12,000 DPI. After ultraviolet exposure, the wafers are baked at $95^{\circ} \mathrm{C}$ for $10 \mathrm{~min}$, developed in fresh propylene glycol monomethyl ether acetate (Sigma Aldrich), and rinsed with fresh propylene glycol monomethyl ether acetate, and baked at $95^{\circ} \mathrm{C}$ for 1 minute to remove solvent. The microfluidic devices are fabricated by curing poly(dimethylsiloxane) (10:1 polymer-to-crosslinker ratio) over the photoresist master ${ }^{30}$. The devices are cured in an $65^{\circ} \mathrm{C}$ oven for 2 hours and extracted with a scalpel, and inlet ports are added using a $0.75 \mathrm{~mm}$ biopsy core (World Precision Instruments). The device is bonded to a glass slide using $\mathrm{O}_{2}$ plasma treatment, and channels are treated with Aquapel (PPG Industries) to render them hydrophobic. Finally, the devices are baked at $65^{\circ} \mathrm{C}$ for 20 min to dry the Aquapel before they are ready for use.

\section{Drop-seq and data analysis}

For scRNA-seq analysis of lung fibrosis, bleomycin ( $3 \mathrm{U} / \mathrm{kg}$; Hospira) or water was instilled intratracheally to anesthetized male 129S1 mice, age 10-12 weeks. After 2 weeks mice were sacrificed, and lungs were perfused with PBS and dissociated to a single cell solution in RPMI containing $0.13 \mathrm{U}$ of liberase TM (Roche) and dissociated using gentleMACS (Miltenyi Biotec). Cells were passed through a $70 \mathrm{um}$ and $40 \mathrm{um}$ strainers. The single cell RNA-seq experiment is performed based on the Drop-seq protocol ${ }^{19}$. Briefly, the barcoded Dropseq beads (ChemGenes corporation, MACOSKO-2011-10) and single cell suspension from

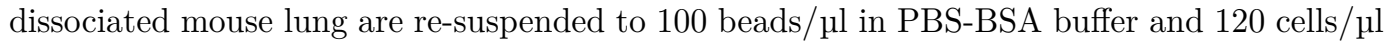
in Drop-seq lysis buffer (with additional $1 \mathrm{M} \mathrm{NaCl}$ added), respectively. Monodisperse droplets $\sim 1 \mathrm{~nL}$ in size were generated using the fabricated Drop-seq device. We used HFE7500 with $2 \% \mathrm{w} / \mathrm{v}$ ionic krytox as oil phase. The collected droplets were broken with perfluorooctanol (Sigma) in $30 \mathrm{ml}$ of $6 \times$ SSC buffer. The beads were then washed and re-suspended in a reverse transcriptase mix for reverse transcription and the template switch reaction. Exonuclease I is used to remove unextended primers following the RT reaction. The beads were then washed, counted, aliquoted into PCR tubes as 6000 beads per PCR reaction, and PCR-amplified for 17 cycles: $95^{\circ} \mathrm{C}$ for $3 \mathrm{~min}$; then 4 cycles of: $98^{\circ} \mathrm{C}$ for $20 \mathrm{sec}$, $65^{\circ} \mathrm{C}$ for $45 \mathrm{sec}, 72^{\circ} \mathrm{C}$ for $3 \mathrm{~min}$; then 13 cycles of: $98^{\circ} \mathrm{C}$ for $20 \mathrm{sec}, 67^{\circ} \mathrm{C}$ for $20 \mathrm{sec}, 72^{\circ} \mathrm{C}$ for $3 \mathrm{~min}$; and finally, $72^{\circ} \mathrm{C}$ for $5 \mathrm{~min}$. The PCR reactions from one Drop-seq run were pooled and purified with AMPure XP beads; the amplified cDNA was quantified with Qubit dsDNA high sensitivity assay and checked on a BioAnalyzer High Sensitivity Chip (Agilent). The 
cDNA was fragmented and amplified for sequencing with the Nextera XT DNA sample prep kit (Illumina) using a primer that enabled the specific amplification of only the 3' ends and Illumina index primers N70X. The libraries were purified with Ampure beads, quantified with Quabit dsDNA high sensitivity assay, checked on a BioAnalyzer High Sensitivity Chip, and then sequenced on Illumina Miseq or Hiseq2000.

Paired-end sequence reads were processed mostly as previously described ${ }^{19}$. Briefly, cell barcode and UMI are extracted from Read 1. Read 2 was aligned to the mouse mm10 genome (UCSC) using Bowtie ${ }^{31}$. Reads mapping to exonic regions of genes of mouse mm10 genome were recorded. The number of transcripts for a given gene within a cell barcode was determined by counting unique UMIs and were compiled into a digital gene expression (DGE) matrix.

\section{Public gene expression data}

Immunological Genome Project (ImmGen) raw expression data of phase 1 and 2 were downloaded as CEL files from GEO (GSE15907 and GSE37448), processed and normalized using the Robust Multi-array Average (RMA) procedure on probe-level data using Matlab functions. The analysis was performed using custom CDF file obtained from Brainarray ${ }^{32}$. Raw counts from of the lung reference dataset were obtained from Gibbins et al. ${ }^{25}$ (GSE94135) and Altboum et al. ${ }^{24}$ (GSE49932). Counts were normalized and converted to TPM using R functions. Gene expression profile of GM-CSF derived bone marrow dendritic cell subsets (available at GSE62361) ${ }^{15}$ was analyzed using the GEO2R tool for differentially expressed genes. Top 50 upregulated by fold-change of GM-DCs genes and top 50 GM-Macs were used in Figure 2b.

\section{Cell Type Annotation of Single Cells}

Feature counts from 10 batches were combined $(42,000$ cells $)$ and analyzed with the Seurat toolkit v2.2 ${ }^{19}$. Cells with less than 500 were filtered out; genes expressed in only 1 cell were omitted. Altogether, the filtered data contained 8,366 cells and 13,861 genes (Table S1). Expression data was log-normalized, and data were scaled to regress out differences in number of detected molecules. Variable genes were identified using the FindVariableGenes function with a x.low.cutoff $=0.0125$, x.high.cutoff $=3$ and y.cutoff $=0.5$. t-SNE plot was computed using the top 10 principal components. SingleR was run in 'DE' mode, choosing reference variable genes based on pairwise cell type differences in expression levels. For each single cell transcriptome, Spearman coefficients for correlations with multiple samples of the same cell type in the reference dataset were computed and separately binned by cell type. Annotation scores of the single cell for each cell type were the recorded as the $75^{\text {th }}$ percentile Spearman coefficient value for each cell type.

For each single-cell, top associated cell types (with $<0.05$ difference from maximal score) were then reanalyzed using only variable genes among those cell types. In each round, the cell type with the lowest correlation or cell types with $>0.05$ difference from maximal score were dropped. This process was iterated until a single cell type remained, which was rendered cell type annotation. More details about the method can be found in Supplementary Information. 
Clustering in Figure 2b was performed using Ward's hierarchical agglomerative clustering method on the SingleR scores across all cell types. Deconvolution analysis in Figure 2a was performed using the DeconRNAseq package ${ }^{33}$ using the average expression of AM samples and average expression of IM3 samples from GSE94135. Differential expression analysis (Figure 2c) was performed using the Seurat package v2.2 with default parameters.

\section{Single cell dissociation for FACS}

Lungs were perfused with PBS and dissociated to a single cell solution in 10mM HEPES RPMI containing $0.2 \%$ collagenase (Wako Pure Chemical Industries), $0.1 \mathrm{mg} / \mathrm{mL}$ Dispase II (Roche), and $2000 \mathrm{U} / \mathrm{mL}$ DNase I (Merck). Cells were passed through a $70 \mathrm{um}$ strainer and stained at $4{ }^{\circ} \mathrm{C}$ for 30 minutes with following antibodies (1:100): SiglecF- APC (clone 1 RNM44N, eBioscience), MHCII-APC-Cy7 (clone M5/114.15.2, eBioscience), CD11c-PE (clone HL3, BD Biosciences). Cells were sorted either with BD FACSAria2 or SONY SH800 FACS. Data for analytical flow was analyzed using FlowJo software.

\section{Bulk RNA-seq}

For bulk RNA-seq analysis of lung fibrosis, bleomycin (3 U/kg; Hospira) or water was instilled intratracheally to anesthetized male 129S1 mice, age 10-12 weeks. Cells gated on SiglecF +CD11c+ and sorted on MHCII-high or -low (Supplemental Figure S7) were collected at 2 weeks and 4 weeks after injury, or at baseline for the MHC-low compartment. RNA was collected (RNeasy Micro, Qiagen) from these samples, and total RNA quality was assessed by spectrophotometer (NanoDrop, Thermo Fisher Scientific Inc., Waltham, MA) and the Agilent 2100 Bioanalyzer (Agilent Technologies, Palo Alto, CA). Intact mRNA was isolated using the Dynabead mRNA Purification Kit for total RNA, according to manufacturer's protocol (Thermo Fisher Scientific, Waltham, MA). Amplified cDNA was prepared using the NuGen Ovation RNA-Seq system V2 kit, according to the manufacturer's protocol (NuGen Technologies, Inc., San Carlos, CA), and sequencing libraries were generated using the Nextera XT library preparation kit with multiplexing primers according to manufacturer's protocol (Illumina, San Diego, CA). Library fragment size distributions were assessed using the Bioanalyzer 2100 and the DNA high-sensitivity chip (Agilent Technologies, Santa Clara, CA). Library sequence quality was assessed by sequencing single-end 50 base pair reads using the Illumina MiSeq platform and were pooled for high-throughput sequencing on the Illumina HiSeq 4000 by using equal numbers of uniquely mapped protein coding reads. RNA sequencing was performed on HiSeq 4000 machines (Illumina, San Diego, CA), followed by de-multiplexing of raw sequencing results, trimming of adapter sequences, and alignment to reference genome using STAR software ${ }^{34}$. DESeq2 was used to normalize by size factor (reads per sample) as well as by library complexity and then applied Wald test to determine significance of differential expression.

\section{Immunostaining and Fluorescence Microscopy}

For immunostaining mouse lungs were perfused with PBS, inflated with 50\% OCT, $10 \%$ sucrose in PBS and fixed overnight at $4 \mathrm{C}$ in $4 \%$ PFA. For human samples OCT embedded 
tissues were cut to $15 \mathrm{um}$ sections and sections were fixed with $4 \%$ PFA for 15 minutes at RT. OCT embedded tissues were cut to $15 \mathrm{um}$ sections, blocked with $3 \%$ donkey serum, $1 \%$ BSA, $0.3 \%$ TritonX and then stained with following antibodies (1:100): SiglecF (clone 1RNM44N, eBioscience or AF1706, R\&D Systems), Mertk (AF591, R\&D Systems), PDGFRb (clone APB5, eBioscience), CD68 (KP1, Abcam or PA1518, Boster), MafB (HPA005653, Sigma). Secondary antibodies conjugated to Alexa fluorophores (Invitrogen and Abcam) were used at concentration 1:200. Stained sections and 2-photon images of second harmonic signal were visualized at Zeiss LSM 780 NLO microscope. Image processing was done using ImageJ.

\section{Fibroblast Migration Assay}

3T3 mouse fibroblasts were seeded at 100\% confluency into a 96-well plate central cell-free detection zone in the center of each well (Platypus technologies). $100 \mu \mathrm{l}$ of conditioned media of macrophage cultures (48h culture in RPMI containing 10\% FBS) were added per well. PDGF-AA antibody (07-1436, Millipore) was added at concentration $0.05 \mathrm{\mu g} / \mathrm{ml}$. Cells were imaged via bright field microscopy at time points: $0 \mathrm{~h}$ and $24 \mathrm{~h}$. Image processing and migration quantification was done using ImageJ.

\section{Measurement of hydroxyproline}

Lung hydroxyproline content was measured as previously described ${ }^{6}$ three weeks after intratracheal instillation of bleomycin to sex-matched, male and female, 8-12-week-old C57BL/6J mice. Briefly, mouse lungs were incubated in $12 \mathrm{~N} \mathrm{HCl}$ at $110^{\circ} \mathrm{C}$ for $18 \mathrm{~h}$. Aliquots of the samples reconstituted in distilled water were added to $1.4 \%$ chloramine- $\mathrm{T}$ in $10 \%$ isopropanol and $0.5 \mathrm{M}$ sodium acetate. Erlich's solution was added, and the samples were incubated at $60^{\circ} \mathrm{C}$ for $10 \mathrm{~min}$. Absorbance at $562 \mathrm{~nm}$ was measured and adjusted according to standard curves.

\section{Human Lung Tissues}

Written, informed, consent was obtained from all subjects and the study approved by the UCSF institutional review board. Pulmonary fibrosis lung tissues were obtained at the time of lung transplantation from patients with a pathologic diagnosis of usual interstitial pneumonia and a consensus clinical diagnosis of idiopathic pulmonary fibrosis (IPF) according to available guidelines ${ }^{35}$. Age-similar, non-diseased, normal lung tissues were procured from lungs not used by the Northern California Transplant Donor Network. Our studies indicate that these lungs are physiologically and pathologically normal ${ }^{36}$. Lung fragments were inflated with $30 \%$ OCT suspended in PBS, embedded in OCT compound and frozen immediately after isolation in dry ice before storing at $-80{ }^{\circ} \mathrm{C}$ until use.

\section{Analysis of published microarray datasets}

Yang et al..$^{29}$ performed gene expression profiling for 167 lung tissues from fibrosing idiopathic interstitial pneumonia subjects and 50 healthy controls. We downloaded raw CEL files GEO 
(Accession GSE32537) and processed using custom CDFs from BrainArray. Normalization was performed using the Robust Multi-array Average (RMA) procedure on Affymetrix microarray data. Single-sample gene set enrichment analysis (ssGSEA) ${ }^{37}$ was performed using human orthologues of $\mathrm{C} 1$ genes (Extended Data: Table S3) and 15 human MHCII genes (HLA-DMA, HLA-DMB, HLA-DOA, HLA-DOB, HLA-DPA1, HLA-DPB1, HLA-DPB2, HLA-DQA1, HLA-DQA2, HLA-DQB1, HLA-DQB2, HLA-DRA, HLA-DRB1, HLA-DRB5, HLA-DRB6).

\section{Statistical analysis}

All significance tests in this paper, unless otherwise stated, were assessed using the two-sided Wilcoxon rank-sum test.

\section{Data Availability}

Raw reads and processed data of single-cell RNA-seq experiments were deposited to GEO, accession GSE111664 (https://www.ncbi.nlm.nih.gov/geo/query/acc.cgi?acc=GSE111664). Bulk RNA-seq expression profiles of SiglecF $+\mathrm{Cd} 11 \mathrm{c}+$ lung macrophages were deposited to GEO, accession GSE111690 (https://www.ncbi.nlm.nih.gov/geo/query/acc.cgi?acc= GSE111690). The SingleR package and R scripts for producing the figures are available at https://github.com/dviraran/SingleR (under the GPL 3.0 license).

\section{References}

1. Rockey, D. C., Bell, P. D. \& Hill, J. A. Fibrosis--A Common Pathway to Organ Injury and Failure. N Engl J Med 373, 96, doi:10.1056/NEJMc1504848 (2015).

2. Blackwell, T. S. et al. Future directions in idiopathic pulmonary fibrosis research. An NHLBI workshop report. Am J Respir Crit Care Med 189, 214-222, doi:10.1164/rccm.2013061141WS (2014).

3. Rock, J. R. et al. Multiple stromal populations contribute to pulmonary fibrosis without evidence for epithelial to mesenchymal transition. Proc Natl Acad Sci U S A 108, E1475-1483, doi:10.1073/pnas.1117988108 (2011).

4. El Agha, E. et al. Two-Way Conversion between Lipogenic and Myogenic Fibroblastic Phenotypes Marks the Progression and Resolution of Lung Fibrosis. Cell Stem Cell 20, 261-273 e263, doi:10.1016/j.stem.2016.10.004 (2017). 5. Hung, C. et al. Role of lung pericytes and resident fibroblasts in the pathogenesis of pulmonary fibrosis. Am J Respir Crit Care Med 188, 820-830, doi:10.1164/rccm.201212-2297OC (2013).

6. Henderson, N. C. et al. Targeting of alphav integrin identifies a core molecular pathway that regulates fibrosis in several organs. Nat Med 19, 1617-1624, doi:10.1038/nm.3282 (2013).

7. Schneider, C. et al. Induction of the nuclear receptor PPAR-gamma by the cytokine GM-CSF is critical for the differentiation of fetal monocytes into alveolar macrophages. Nat Immunol 15, 1026-1037, doi:10.1038/ni.3005 (2014). 
8. Tan, S. Y. \& Krasnow, M. A. Developmental origin of lung macrophage diversity. Development 143, 1318-1327, doi:10.1242/dev.129122 (2016).

9. Gibbons, M. A. et al. Ly6Chi monocytes direct alternatively activated profibrotic macrophage regulation of lung fibrosis. Am J Respir Crit Care Med 184, 569-581, doi:10.1164/rccm.201010-1719OC (2011).

10. Misharin, A. V. et al. Monocyte-derived alveolar macrophages drive lung fibrosis and persist in the lung over the life span. J Exp Med 214, 2387-2404, doi:10.1084/jem.20162152 (2017).

11. Hashimshony, T. et al. CEL-Seq2: sensitive highly-multiplexed single-cell RNA-Seq. Genome Biol 17, 77, doi:10.1186/s13059-016-0938-8 (2016).

12. Heng, T. S., Painter, M. W. \& Immunological Genome Project, C. The Immunological Genome Project: networks of gene expression in immune cells. Nat Immunol 9, 1091-1094, doi:10.1038/ni1008-1091 (2008).

13. Amit, I. et al. Unbiased reconstruction of a mammalian transcriptional network mediating pathogen responses. Science 326, 257-263, doi:10.1126/science.1179050 (2009).

14. Shalek, A. K. et al. Single-cell RNA-seq reveals dynamic paracrine control of cellular variation. Nature 510, 363-369, doi:10.1038/nature13437 (2014).

15. Helft, J. et al. GM-CSF Mouse Bone Marrow Cultures Comprise a Heterogeneous Population of CD11c(+)MHCII(+) Macrophages and Dendritic Cells. Immunity 42, 11971211, doi:10.1016/j.immuni.2015.05.018 (2015).

16. Guilliams, M. \& Malissen, B. A Death Notice for In-Vitro-Generated GM-CSF Dendritic Cells? Immunity 42, 988-990, doi:10.1016/j.immuni.2015.05.020 (2015).

17. Li, H. et al. Reference component analysis of single-cell transcriptomes elucidates cellular heterogeneity in human colorectal tumors. Nat Genet 49, 708-718, doi:10.1038/ng.3818 (2017).

18. Kang, H. M. et al. Multiplexed droplet single-cell RNA-sequencing using natural genetic variation. Nat Biotechnol 36, 89-94, doi:10.1038/nbt.4042 (2018). 19. Macosko, E. Z. et al. Highly Parallel Genome-wide Expression Profiling of Individual Cells Using Nanoliter Droplets. Cell 161, 1202-1214, doi:10.1016/j.cell.2015.05.002 (2015).

20. Satija, R., Farrell, J. A., Gennert, D., Schier, A. F. \& Regev, A. Spatial reconstruction of single-cell gene expression data. Nat Biotechnol 33, 495-502, doi:10.1038/nbt.3192 (2015).

21. Gautier, E. L. et al. Gene-expression profiles and transcriptional regulatory pathways that underlie the identity and diversity of mouse tissue macrophages. Nat Immunol 13, 1118-1128, doi:10.1038/ni.2419 (2012).

22. Hume, D. A., Mabbott, N., Raza, S. \& Freeman, T. C. Can DCs be distinguished from macrophages by molecular signatures? Nat Immunol 14, 187-189, doi:10.1038/ni.2516 (2013).

23. Randolph, G. \& Merad, M. Reply to: "Can DCs be distinguished from macrophages by molecular signatures?". Nat Immunol 14, 189-190, doi:10.1038/ni.2517 (2013). 
24. Altboum, Z. et al. Digital cell quantification identifies global immune cell dynamics during influenza infection. Mol Syst Biol 10, 720, doi:10.1002/msb.134947 (2014).

25. Gibbings, S. L. et al. Three Unique Interstitial Macrophages in the Murine Lung at Steady State. Am J Respir Cell Mol Biol 57, 66-76, doi:10.1165/rcmb.2016-0361OC (2017).

26. Ishida, Y. et al. Essential involvement of the CX3CL1-CX3CR1 axis in bleomycin-induced pulmonary fibrosis via regulation of fibrocyte and M2 macrophage migration. Sci Rep 7, 16833, doi:10.1038/s41598-017-17007-8 (2017).

27. Yona, S. et al. Fate mapping reveals origins and dynamics of monocytes and tissue macrophages under homeostasis. Immunity 38, 79-91, doi:10.1016/j.immuni.2012.12.001 (2013).

28. Zhou, X. et al. Circuit Design Features of a Stable Two-Cell System. Cell 172, 744-757 e717, doi:10.1016/j.cell.2018.01.015 (2018).

29. Yang, I. V. et al. Expression of cilium-associated genes defines novel molecular subtypes of idiopathic pulmonary fibrosis. Thorax 68, 1114-1121, doi:10.1136/thoraxjnl-2012-202943 (2013).

30. Qin, D., Xia, Y. \& Whitesides, G. M. Soft lithography for micro- and nanoscale patterning. Nature protocols 5, 491-502, doi:10.1038/nprot.2009.234 (2010).

31. Langmead, B., Trapnell, C., Pop, M. \& Salzberg, S. L. Ultrafast and memory-efficient alignment of short DNA sequences to the human genome. Genome Biology 10, 1-10, doi:10.1186/gb-2009-10-3-r25 (2009).

32. Dai, M. et al. Evolving gene/transcript definitions significantly alter the interpretation of GeneChip data. Nucleic Acids Res 33, e175, doi:10.1093/nar/gni179 (2005).

33. Gong, T. \& Szustakowski, J. D. DeconRNASeq: a statistical framework for deconvolution of heterogeneous tissue samples based on mRNA-Seq data. Bioinformatics 29, 1083-1085, doi:10.1093/bioinformatics/btt090 (2013).

34. Dobin, A. et al. STAR: ultrafast universal RNA-seq aligner. Bioinformatics 29, 15-21, doi:10.1093/bioinformatics/bts635 (2013).

35. Raghu, G. et al. An official ATS/ERS/JRS/ALAT statement: idiopathic pulmonary fibrosis: evidence-based guidelines for diagnosis and management. Am J Respir Crit Care Med 183, 788-824, doi:10.1164/rccm.2009-040GL (2011).

36. Ware, L. B. et al. Assessment of lungs rejected for transplantation and implications for donor selection. Lancet 360, 619-620 (2002).

37. Verhaak, R. G. et al. Prognostically relevant gene signatures of high-grade serous ovarian carcinoma. J Clin Invest 123, 517-525, doi:10.1172/JCI65833 (2013).

\section{Acknowledgements}

This work was supported by a UCSF Marcus Award to M.B. and A.A., a National Institutes of Health grant (HL131560) to M.B, a Gruss Lipper Postdoctoral Fellowship to D.A., and the National Institute of Allergy and Infectious Diseases (Bioinformatics Support Contract HHSN272201200028C) to A.J.B. The content is solely the responsibility of the authors and 
does not necessarily represent the official views of the National Institutes of Health. We thank D. Erle, A. Barczak, W. Eckalbar, and M Adkisson of the UCSF Functional Genomics Core Facility and D. Sheppard for his insightful comments on the manuscript.

\section{Author Contributions}

D.A. developed the cell type annotation tool presented and performed computational analysis of single cell data under the guidance of A.B; A.L. performed in vivo and in vitro experiments on macrophage lineage and function under the guidance of M.B; L.L. performed microfluidic capture of single cell transcriptomes, library preparation, and sequencing under the guidance of A.A; V.F. and A.H prepared breeding and experimental stocks of genetically modified mice and performed lung injury models under the guidance of M.B.; P.J.W. contributed acquisition, storage, and processing of human samples; M.B. conceived of the work, supervised experimental planning and execution, and wrote the manuscript with input from D.A., A.L., and L.L.

\section{Competing interests}

The authors declare no competing financial interest. 